\title{
Regulation of low-density lipoprotein receptor and 3-hydroxy-3- methylglutaryl coenzyme a reductase gene expression by thymoquinone-rich fraction and thymoquinone in HepG2 cells.
}

\begin{abstract}
Background and Aim: Nigella sativa and its active constituent thymoquinone (TQ) have been exploited for their various health benefits. This work was aimed to investigate the regulatory effects of TQ-rich fraction (TQRF) and commercial TQ on the low-density lipoprotein receptor (LDLR) and 3-hydroxy-3- methylglutaryl-coenzyme A reductase (HMGCR) genes in HepG2 cells. Methods and Results: TQRF was extracted from N. sativa seeds using supercritical fluid extraction. The regulatory effects of TQRF at $80 \mu \mathrm{g} / \mathrm{ml}$ and TQ at $2 \mu \mathrm{g} / \mathrm{ml}$ on LDLR and HMGCR gene expression were investigated in HepG2 cells using quantitative real-time PCR. The TQ content in TQRF was $2.77 \%(\mathrm{w} / \mathrm{w})$ and was obtained at a temperature of $40^{\circ} \mathrm{C}$ and a pressure of 600 bar. Treatment of cells with TQRF and TQ resulted in a 7- and 2-fold upregulation of LDLR mRNA level, respectively, compared with untreated cells. The mRNA level of HMGCR was downregulated by 71 and $12 \%$, respectively, compared with untreated cells. Conclusion: TQRF and TQ regulated genes involved in cholesterol metabolism by two mechanisms, the uptake of low-density lipoprotein cholesterol via the upregulation of the LDLR gene and inhibition of cholesterol synthesis via the suppression of the HMGCR gene.
\end{abstract}

Keyword: 25-Hydroxycholesterol; 3-Hydroxy-3-methyl-glutaryl-coenzyme A reductase; HepG2 cells; Low-density lipoprotein receptor; Nigella sativa; Supercritical fluid extraction; Thymoquinone; Thymoquinone-rich fraction. 